Journal of Universal Language 3

September 2002, 31-56

\title{
Typology of Coreferential Possessive Anaphora and Neo-Gricean Pragmatics: Implications for a Newly Designed Artificial Language
}

\author{
Yan Huang \\ University of Reading
}

\begin{abstract}
Coreferential possessive anaphora can be defined as a relation between two linguistic expressions, one a possessive anaphor, and the other its antecedent, in which the possessive anaphor refers to what its antecedent refers to. This article presents a typology of coreferential possessive anaphora, on the basis of a preliminary investigation into a variety of genetically different and structurally distinct languages in the world. It also provides an analysis of coreferential possessive anaphora in terms of the revised neo-Gricean pragmatic theory of anaphora, as constructed in Huang (2000a). Finally, coreferential possessive anaphora in Esparento will be examined, and implications for the construction of such anaphora in a newly designed artificial language will be discussed.
\end{abstract}

Keywords: possessive anaphora, typology, neo-Gricean pragmatics, natural languages, artificial languages 


\section{Introduction}

Coreferential possessive anaphora can be defined as a relation between two linguistic expressions, one a possessive anaphor, and the other its antecedent, in which the possessive anaphor refers to what its antecedent refers to, as can be shown by (1) below. ${ }^{1}$

(1) $(\text { Chinese })^{2}$

Xiaohua $_{1}$ xihuan $\mathrm{ziji}_{1} / \mathrm{ta}_{1 / 2}$ de youeryuan.

Xiaohua like self/3.Sg of nursery

"Xiaohua ${ }_{1}$ likes self's ${ }_{1} /$ his $_{1 / 2}$ nursery."

In (1), ziji/ta is the possessive anaphor, and Xiaohua is its antecedent. The possessive anaphor and its antecedent are coreferential with each other. ${ }^{3}$

In Huang (2000a), I discussed coreferential possessive anaphora,

${ }^{1}$ I am grateful to the anonymous reviewers, of the Journal of Universal Language for their useful comments on the earlier versions of this article. Thanks also go to John Wells and Jardar Abrahamsen for their help with the Esparento data.

2 The following abbreviations will be used in glosses of examples: Abs, absolutive; Acc, accusative; Arg, agreement; Aux, auxiliary; Cl, classifier; Dat, dative; Emph, emphatic; Erg, ergative; Gen, genitive; M, masculine; Nom, nominative; Pst, past tense; Poss, possessive; Refl, reflexive; Subj, subject; Top, topic; $1 \mathrm{Sg}$, first-person singular personal pronoun; $3 \mathrm{Sg}$, third-person singular personal pronoun; 3P, third-person plural personal pronoun.

3 In some languages, an r-expression such as a proper name occasionally may also be used as a coreferential possessive anaphora, as can be exemplifed by (1) below.

(1) Chinese

Xiaohua $_{1}$ xihua Xiaohua $_{1}$ de youeryuan.

Xiaohua like Xiaohua of nursery

"Xiaohua ${ }_{1}$ likes Xiaohua's nursery."

But in this article, I shall restrict myself to those coreferential possessive anaphors that are encoded in possessive reflexives and pronouns. 
but the space at my disposal there did not allow me to give it a detailed analysis. The aim of this article is threefold: firstly to present a typology of coreferential possessive anaphora, on the basis of a preliminary investigation into a variety of genetically different and structurally distinct languages in the world, secondly to provide an analysis of coreferential possessive anaphora in terms of the revised neo-Gricean pragmatic theory of anaphora, as constructed in Huang (2000a) (see also Levinson 2000), and thirdly to discuss coreferential possessive anaphora in Esparento and propose a model for setting up such anaphora in a newly constructed artificial language. The article is organised as follows. In section 2, I shall present a typology of coreferential possessive anaphora, Next, I shall outline the three Levinsonian neo-Gricean pragmatic principles in subsection 3.1 , and the revised neo-Gricean pragmatic theory of anaphora in subsection 3.2. Then, in subsection 3.3, I shall provide a revised neo-Gricean pragmatic analysis of coreferential possessive anaphora. Finally, in section 4, I shall discuss coreferential possessive anaphora in Esparento and the implications for the construction of such anaphora in a newly designed artificial language.

\section{A Typology of Coreferential Possessive Anaphora}

With respect to the reflexive/pronoun distribution in coreferential possessive anaphora, languages can be divided into three types, following in part a suggestion by Burzio (1996) (see also Huang 2000a: 24-25): (i) those allowing reflexives but not pronouns, (ii) those permitting pronouns but not reflexives, and (iii) those warranting both reflexives and pronouns. In the first type, the possessive and the antecedent are 'close' enough to allow only a reflexive but not a pronoun for a coreferential interpretation. Basque, Ingush and Telugu ((2)-(4)) are, for instance, examples of languages of this type. 
Let us call this type 'reflexives only' languages.

(2) (Basque, Rebuschi 1987)

Peiok $_{1} \quad$ bere $_{1} /$ haren $_{1}$ txakurra ikusi du.

Peio self's/his dog seen Aux

"Peio ${ }_{1}$ has seen self's , $_{1}$ his $*$ dog."

(3) (Ingush, Nicholas 2001)

Muusaaz $_{1} \quad$ shii $_{1} / \mathrm{cyn}_{2} \quad$ bierazhta

Muusaaz-Erg 3SG-Refl-Gen/3Sg-Gen children-Dat

kinashjka icaad.

book bought

"Musa bought self's $_{1} /$ his $_{2}$ children a book."

(4) (Telugu, Subbarao \& Lalitha Murthy 2000: 238, 255)

roojaa-ki $\operatorname{tana}_{1} /$ atani $_{2}$ amma anTee iSTam.

Raja-Dat self's/his mother means liking

"Roja ${ }_{1}$ likes self's ${ }_{1} /$ his $_{2}$ mother."

Next in the second category, because either there is no possessive reflexive in the language or the possessive reflexive cannot be used, only a pronoun is permitted for a coreferential reading. Akan, English and Guugu Yimidhirr ((5)-(7)), for example, belong to this type. Let us dub this type 'pronouns only' languages.

(5) (Akan, Faltz 1985)

$\mathrm{John}_{1}$ praa $\mathrm{ne}_{1 / 2} \quad$ 'fie.

John swept 3Sg-Poss house

"John swept his $_{1 / 2}$ house."

(6) (English)

David $_{1}$ has lent his ${ }_{1 / 2}$ Picasso to a museum. 
(7) (Guugu Yimidhirr, Levinson 1987)

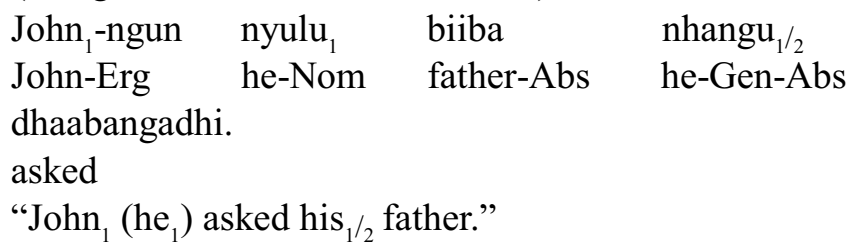

Finally in the third group, the possessive and the antecedent are both 'close' enough to allow a reflexive and at the same time 'distant' enough to permit a pronoun as well to encode coreferentiality. Examples provided for this type include those of Korean, Oriya and Tuki ((8)-(10)). Let us term this type 'both reflexives and pronouns' languages. ${ }^{4}$

(8) (Korean)

$\mathrm{Kim}_{1}$-un caki $_{1} / \mathrm{ku}_{-} / 2$ uy emma-lul hyemohanta.

Kim-Top self/his-Gen mom-Acc hate

"Kim 1 hates self's $/$ his $_{1 / 2}$ mom."

(9) (Oriya, Ray 2000)

4 There may be a mixture of the types within a language. For example, Mundani is a 'reflexives only' language in third person singular, but a 'pronouns only' language in third person plural, as can be seen from the following examples (data from Parker 1986).

(1) ta ${ }_{1}$ dzí akèndè á-zí ${ }_{1} /$ tò $_{2}$. $3 \mathrm{Sg}$ eat banana self's/his

"He ${ }_{1}$ has eaten self's ${ }_{1} /$ his $_{2}$ banana."

(2) $b^{\supset}$ le ni eghi $\quad b^{\supset} b_{1 / 2}$.

They 3P1 take things their

"They ${ }_{1}$ took their ${ }_{1 / 2}$ things." 
36 Typology of Coreferential Possessive Anaphora and Neo-Gricean Pragmatics

raama $_{1}$ nija $_{1} /$ taa $_{1}$ bahi paDhilaa.

Rama self's his book read

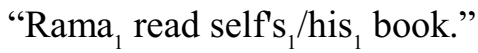

(10) (Tuki, Biloa 1991)

Mbara $_{1}$ a mu kusa vakarate vaamate $_{1} /$ vaa $_{1}$. Mbara Sbj Pl buy books 3Sg self/his

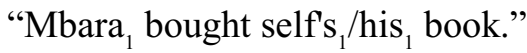

\section{A Revised Neo-Gricean Pragmatic Account of Coreferential Possessive Anaphora}

In the last section, I have presented a typology for coreferential possessive anaphora. In this section, I shall provide an analysis of it in terms of the revised neo-Gricean pragmatic theory of anaphora, as constructed in Huang (2000a) (see also Levinson 2000). I shall begin with a discussion of the three Levinsonian inferential principles in subsection 3.1. I shall then outline the revised neo-Gricean pragmatic theory of anaphora in subsection 3.2. Finally in subsection 3.3, I shall offer a revised neo-Gricean pragmatic account of coreferential possessive anaphora.

\subsection{Inferential Principles in a Neo-Gricean Pragmatic Theory}

On a general Gricean account of meaning and communication, there are two theories: a theory of meaning-n[on]-n[atural] and a theory of conversational implicature (e.g., Grice 1989). In the theory of meaning-nn, Grice (1989) emphasizes the conceptual relation between natural meaning in the external world and non-natural, linguistic meaning of utterances. He develops a reductive analysis of

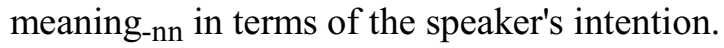

In his theory of conversational implicature, Grice proposes that 
there is an underlying principle that determines the way in which language is used maximally efficiently and effectively to achieve rational interaction in communication. He calls this governing dictum the co-operative principle and subdivides it into nine maxims classified into four categories. The co-operative principle and its component maxims ensure that in an exchange of conversation, the right amount of information is provided and that the interaction is conducted in a truthful, relevant, and perspicuous manner.

One recent advance on the classical Gricean account is the neoGricean pragmatic theory put forward by Levinson (1987, 1991, 2000). Levinson proposes (aside from the irreducible maxim of Quantity) that the original Gricean programme be reduced to three neo-Gricean pragmatic principles, what he dubs the Q[uantity]-, I[nformativeness]-, and M[anner]-principles.

\section{(11) The Q-principle}

a. Speaker's maxim:

Do not provide a statement that is informationally weaker than your knowledge of the world allows, unless providing a stronger statement would contravene the Iprinciple.

b. Recipient's corollary:

Take it that the speaker made the strongest statement consistent with what he knows, and therefore that:

(i) if the speaker asserted $A(W)$, where $A$ is a sentence frame and $W$ an informationally weaker expression than $S$, and the contrastive expressions $<S, W>$ form a Horn scale (in the prototype case, such that $A(S)$ entails $A(W)$ ), then one can infer that the speaker knows that the stronger statement $A(S)$ (with $S$ substituted for $W$ ) would be false (or $K \sim(A(S))$ );

(ii) if the speaker asserted $A(W)$ and $A(W)$ fails to entail an embedded sentence $Q$, which a stronger statement 
$A(S)$ would entail, and $<S, W>$ form a contrast set, then one can infer the speaker does not know whether $Q$ obtains or not (i.e., $\sim K(Q)$ or equally $\{P(Q)$, $P \sim(Q)\})$.

The basic idea of the metalinguistic Q-principle is that the use of an expression (especially a semantically weaker one) in a set of contrastive semantic alternates Q-implicates the negation of the interpretation associated with the use of another expression (especially a semantically stronger one) in the same set. In other words, the effect of this inferential strategy is to give rise to an upper-bounding conversational implicature: from the absence of an informationally stronger expression, we infer that the interpretation associated with the use of that expression does not hold. Using the symbol $+>$ to mean 'conversationally implicate': we can represent the Qimplicature schematically in (12), and exemplify it in (13).

Q-scale: $<x, y>$ $\mathrm{y}+>_{\mathrm{Q}} \sim \mathrm{x}$

(13) $Q_{\text {-scalar: }}$ <all, some $>$

Some of my friends love Christmas carols.

$+>$ Not all of my friends love Christmas carols

In (13) there is a Q-scale <all, some>. In this scale all is semantically stronger than some, because all entails some but not vice versa. If the speaker selects the semantically weaker some, he or she conversationally implies that he or she is not in a position to use the semantically stronger all truth-conditionally, thus the indicated interpretation. Next the I-principle.

(14) The I-principle

a. Speaker's maxim: the maxim of minimization 
'Say as little as necessary', that is, produce the minimal linguistic information sufficient to achieve your communicational ends, (bearing the Q-principle in mind).

b. Recipient's corollary: the rule of enrichment Amplify the informational content of the speaker's utterance, by finding the most specific interpretation, up to what you judge to be the speaker's m-intended point, unless the speaker has broken the maxim of minimization by using a marked or prolix expression.

c. Specifically:

(i) Assume the richest temporal, causal and referential connections between described situations or events, consistent with what is taken for granted.

(ii) Assume that stereotypical relations obtain between referents or events, unless this is inconsistent with (11).

(iii) Avoid interpretations that multiply entities referred to (assume referential parsimony); specifically, prefer coreferential readings of reduced NPs (pronouns or zeros).

(iv) Assume the existence or actuality of what a sentence is about if that is consistent with what is taken for granted.

The central idea of the I-principle is that the use of a semantically general linguistic expression I-implicates a semantically specific interpretation. In other words, the operation of the I-principle induces an inference to a proposition that accords best with the most stereotypical and explanatory expectation given real world knowledge. Schematically:

$$
\begin{aligned}
& \text { (15) I-scale: }[\mathrm{x}, \mathrm{y}] \\
& \mathrm{y}+>_{\mathrm{I}} \mathrm{x}
\end{aligned}
$$


40 Typology of Coreferential Possessive Anaphora and Neo-Gricean Pragmatics

(16) (Conjunction buttressing) $p$ and $q$

$+>p$ and then $q$

$+>p$ and therefore $q$

$+>p$ in order to cause $q$

John pressed the spring and the drawer opened.

$+>$ John first pressed the spring and then the drawer opened

$+>$ John pressed the spring and therefore the drawer opened

$+>$ John pressed the spring in order to cause the drawer to open

In (16), the speaker opts for a semantically general and and by the I-principle we obtain a semantically more specific interpretation such as and then, and therefore or in order to cause. Finally the Mprinciple:

(17) The M-principle

a. Speaker's maxim:

Indicate an abnormal, non-stereotypical situation by using marked expressions that contrast with those you would use to describe the corresponding normal, stereotypical situation.

b. Recipient's corollary:

What is said in an abnormal way indicates an abnormal situation, or marked messages indicate marked situations.

c. Specifically:

Where $\mathrm{S}$ has said $\mathrm{p}$ containing marked expression $\mathrm{M}$, and there is an unmarked alternate expression $U$ with the same denotation D which the speaker might have employed in the same sentence frame instead, then where U would have I-implicated the stereotypical or more spe- 
cific subset $d$ of $D$, the marked expression $M$ will implicate the complement of the denotation $d$, namely $d$ of $D$.

The basic idea of the metalinguistic M-principle is that the use of a marked expression M-implicates the negation of the interpretation associated with the use of an alternative, unmarked expression in the same set. In other words, from the use of a marked expression, we infer that the stereotypical interpretation associated with the use of an alternative, unmarked expression does not hold. Schematically:

(18) M-scale: $\{x, y\}$

$\mathrm{y}+>_{\mathrm{M}} \sim \mathrm{x}$

(19) a. The tram comes frequently

$+>$ The tram comes, say, every ten minutes

b. The tram comes not infrequently

$+>$ The tram comes not as frequently as the uttering of

(a) suggests, say, every twenty minutes

Here, while (19a) is unmarked, (19b) is marked, being a double negation construction. Given the M-principle, if the marked (19b) is used, then a marked interpretation is generated, such as the one indicated above. Thus, taken together, the I-, and M-principles give rise to complementary interpretations: the use of an unmarked expression tends to convey an unmarked message, whereas the use of a marked expression tends to convey a marked message (see e.g., Huang 1991, 1994, 2000a, 2000b, and in preparation for further discussion).

\subsection{A Revised Neo-Gricean Pragmatic Theory of Anaphora}

Having briefly discussed the three Levinsonian neo-Gricean 
pragmatic principles, I now proceed to outline a revised neo-Gricean pragmatic theory of anaphora, based on Huang (1991, 1994, 1995, 1996, 2000a, 2000b) and Levinson (1987, 1989, 2000).

The central idea underlying the revised neo-Gricean pragmatic theory is that the interpretation of certain patterns of anaphora can be made utilizing pragmatic inferences, dependent on the language user's knowledge of the range of options available in the grammar, and of the systematic use or avoidance of particular anaphoric expressions or structures on particular occasions.

Applying the Q-, I-, and M-principles, sketched in subsection 3.1 above, to the domain of anaphoric reference, we can derive a revised neo-Gricean pragmatic apparatus for the interpretation of various types of anaphoric expression. Assuming the hierarchy of referentiality for different kinds of anaphoric expression in (20), along the lines of Burzio (1991, 1996), Levinson (1991, 2000), and Huang (1991, 1994, 2000a), this pragmatic apparatus can be presented in (21).

(20) A hierarchy of referentiality for different types of anaphoric expression

Anaphors $<$ pronominals $<$ r-expressions (Anaphors (i.e., reflexives and reciprocals) are less referential than pronominals (e.g., pronouns), and pronominals are less referential than r-expressions (e.g., proper names and definite descriptions).)

(21) A revised neo-Gricean pragmatic apparatus for anaphora (Huang 2000a)

a. Interpretation principles

(i) The use of an anaphoric expression $x$ I-implicates a local coreferential interpretation, unless (ii) or (iii).

(ii) There is an anaphoric Q-scale $<x, y>$, where informally $x$ semantically stronger than $y$, in which case, 
the use of y Q-implicates the complement of the Iimplicature associated with the use of $\mathrm{x}$, in terms of reference.

(iii) There is an anaphoric $M$-scale $\{x, y\}$, where informally $\mathrm{x}$ is unmarked with respect to, or simpler than $\mathrm{y}$, in which case, the use of y M-implicates the complement of the I-implicature associated with the use of $\mathrm{x}$, in terms of either reference or expectedness.

b. Consistency constraints

Any interpretation implicated by (a) is subject to the requirement of consistency with

(i) The revised DRP. ${ }^{5}$

(ii) Information saliency, so that

(a) implicatures due to matrix constructions may take precedence over implicatures due to subordinate constructions, and

(b) implicatures to coreference may be preferred according to the saliency of antecedent in line with the hierarchy topic $>$ subject $>$ object, etc.; and

(iii) General implicature constraints, namely,

(a) background assumptions,

(b) contextual factors

(c) meaning-nn, and

(d) semantic entailments.

5 What the revised $\mathrm{D}$ [isjoint]R[eference]P[resumption] states is that the coarguments of a predicate are intended to be disjoint, unless one of them is reflexive-marked (Huang 2000a). Note also that the DRP was originally proposed by Farmer and Harnish (1987), which goes like this: the arguments of a predicate are intended to be disjoint, unless marked otherwise. Since the DRP is irrelevant with regard to coreferential possessive anaphora; the possessive anaphor and its antecedent being non-co-arguments of the same predicate, I shall not discuss it here (but see Huang 2000a for a detailed discussion). 


\subsection{A Revised Neo-Gricean Pragmatic Analysis}

With (20) and (21) in place, we can now give a revised neoGricean pragmatic account of coreferential possessive anaphora. Let me start with the 'reflexives only' type of language. In addition to (2)-(4) above, there are (22)-(27).

(22) (Gimira, Breeze 1986)

$\mathrm{ba} / \mathrm{yi}$ dor gotue.

3-Refl/his sheep sold-3-M-Fin

"He $\mathrm{H}_{1}$ sold self's $\mathrm{h}_{1} / \mathrm{his}_{2}$ sheep."

(23) (Hindi/Urdu, Davison 1997)

Syaam $_{1}$ apniii $_{1} / \mathrm{us}_{2}$ praSaNsaa nahiiN

Shyam self $/ \mathrm{s}^{\prime} 3 \mathrm{Sg}$-of praise do-Impf

kar-taa.

not do-Impf

"Shyam ${ }_{1}$ does not do self's s $_{1}$ his $_{2}$ praise."

(24) (Japanese, Kitagawa 1982: 206) ${ }^{6}$

$\mathrm{Katyoo}_{1}$-ga zibun $/ \mathrm{kare}_{2}$-no hisyo.

section chief-Subj self/his secretary

to kekkon-sita

with married

"The section chief $f_{1}$ married self's / $_{1}$ his $_{2}$ secretary."

(25) (Kashimiri, Wali et al 2000: 484, 494)

\footnotetext{
${ }^{6}$ One or two Japanese native speakers I have consulted think that a coreferential reading is possible between the pronoun and its antecedent in this example. If this is the case, then Japanese may also be a 'both reflexives and pronouns' language. The disjoint reading may be due to the fact that the pronoun is not appropriate for an antecedent whose referent is in a socially higher position (see e.g., Shibatani 1990: 378).
} 
a. Miiraa ${ }_{1}$ cha paunu ${ }_{1}$ gari gatshaan. Mira is self's house go "Mira is going to self's ${ }_{1}$ house."

b. Mohn-an $n_{1}$ dits tcm'sinden $*_{1 / 2}$ doost-an Mohan-Erg gave his-Dat friends-Dat kitaab.

book

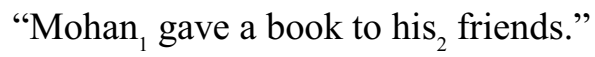

(26) (Latin, Burzio 1996)

Ioannes $_{1}$ sororem suam $_{1} /$ eius $_{1}$ vidit. Ioannes sister self's/his saw "Ioannes ${ }_{1}$ saw self /his $_{2}$ sister."

(27) (Norwegian, Hestvik 1992)

$\mathrm{John}_{1}$ fortalte $\mathrm{Ola}_{2}$ om $\sin _{1} /$ hans $_{1}$ kone. John told Ola about Refl/his wife "John 1 told Ola 2 about self $1 /$ his $_{2}$ wife."

Note again that all the languages in (2)-(4) and (22)-(27) have one thing in common: they allow only a reflexive but not a pronoun to encode coreferentiality for possessive anaphora. Given (21) above, the interpretation of the reflexive is subject to the I-principle (you use a more general term, I get a more specific interpretation), which engenders a local coreferential reading. The interpretation of the pronoun is then due to the working of the Q-principle. By the referentiality hierarchy (20) and the I-principle, a reflexive will be chosen if coreference is intended, because the reflexive is referentially most economic. This has the consequence that if the reflexive is not employed but a pronoun is used instead, a classic Q-implicature will arise; namely, no coreference is intended. In other words, we have a Q-scale $<$ reflexive, pronoun $>$ here, such that the use of the semantically weaker pronoun Q-implicates that the use of the semantically 
stronger reflexive cannot be truthfully entertained, that is, the coreferential interpretation which is associated with the use of the reflexive should be avoided. Reflexives are semantically stronger than pronouns in that (i) syntactically, they typically need to be bound in some domain, and (ii) semantically, they are normally referentially dependent.

Going next to the 'pronouns only' type of language, consider (5)(7) above and (28)-(30) below.

(28) (Arabic)

As'ad $_{1}$ daxala maktab-hu ${ }_{1 / 2}$.

Asad enter-Pst-3Msg office-his

"Asad entered his $_{1 / 2}$ office."

(29) (German)

Ekkehard $_{1}$ sah sein $_{1 / 2}$ Buck.

Ekkehard saw his book

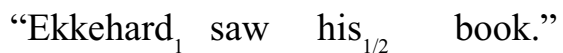

(30) (Spanish)

Maria $_{1}$ quiere a $\operatorname{sus}_{1 / 2}$ amigos.

Mary loves Acc 3-Poss friends

"Mary ${ }_{1}$ loves her ${ }_{1 / 2}$ friends."

In these cases, since no reflexive is available as a possible candidate to indicate coreferentiality, by the referentiality hierarchy (20), a pronoun is used instead. As a result, there is no Q-scale $<$ reflexive, pronoun $>$ to prevent the pronoun falling under the I-principle, which gives rise to a preferred local coreferential interpretation. Note that unlike some generative analyses such as Chomsky's $(1981,1995)$ binding theory, the revised neo-Gricean pragmatic account being advocated here can successfully account for those coreferential possessive anaphora patterns where a pronoun is happily bound in its 
local domain. This is because given the referentiality hierarchy (20), the pronoun has now become the most favoured choice for encoding coreferentiality. Thus, our revised neo-Gricean pragmatic theory correctly allows coreferentiality to be marked by a lower ranked anaphoric expression (such as a pronoun) if its immediately higher ranked counterpart (such as a reflexive) is not available - an analysis that is empirically more accurate.

Finally, let us move to the 'both reflexives and pronouns' type of language. We have already seen examples from Chinese, Oriya, and Tuki in (8)-(10) above. Further examples from a variety of other languages are give in (31)-(36) below.

(31) (Bangala, Sengupta 2000: 291, 299)
a. babli
nije-r ${ }_{1} \quad$ kaj
korche.
Babli-Nom self's work-Acc is doing "Babli is doing self's, work."
$\begin{array}{lllll}\text { b. }{ }^{\mathrm{D}} \text { Yon } & \text { ta-r } & \text { boy-Ta } & \text { ama-ke } & \text { dilo. } \\ \text { Nayan-Nom } & \text { his } & \text { book-Acc } & \text { 1Sg-dat } & \text { gave }\end{array}$ "Nayan gave his book to me."

(32) (Kannada, Amritavalli 2000: 62, 75)

Raama $_{1}$ tanna /avana $_{1}$ makkaLanna-ho Dedanu. Rama self's children-Acc beat "Rama beat self's $_{1} /$ his $_{1}$ children."

(33) (Malay, cited in Huang 2000) Aminah $_{1}$ mencuci pakaian-dirinya ${ }_{1} /$ nya $_{1 / 2}$. Aminah wash clothes-self her/her "Aminah washes herself's $_{1} /$ her $_{1 / 2}$ clothes." 
(34) (Malayalam, Mohanan 1982)

Moohan $_{1}$ tante $_{1} /$ awante $_{1}$ bhaaryaye nulli.

Mohan self's his wife pinched

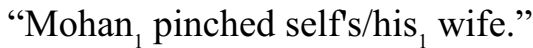

(35) (Marathi, Wali 2000: 527, 547)

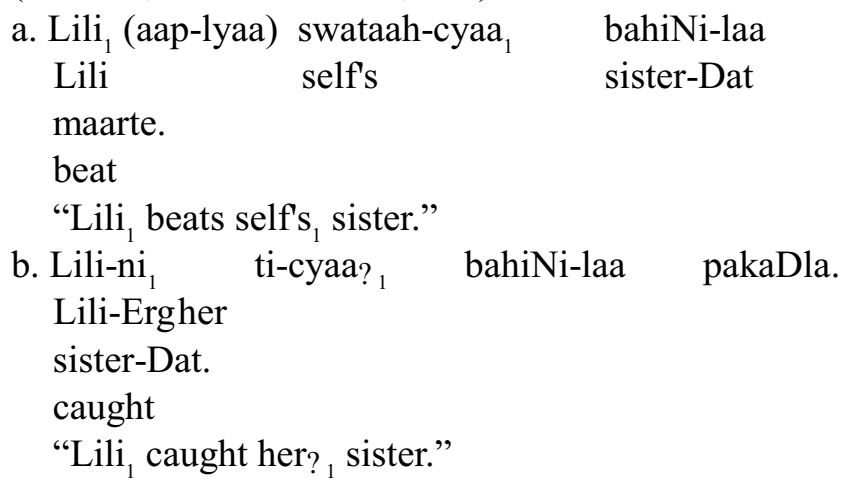

(36) (Tamil, Annamalai 2000)

kumaar $_{1} \tan _{1} / \operatorname{avan}_{1 / 2}$ tambiyeyee

Kumar self/he brother-Acc-Emph

verukraan.

hate-Prst-Agr

"Kumar ${ }_{1}$ hates self's si/2 $_{1}$ his brother."

This type of coreferential possessive anaphora is of particular interest to us, for there is a distributional overlap between the reflexive and the pronoun. Given the referentiality hierarchy (20), one immediate question arises: why should there be such an overlap? One plausible view, due to Burzio (1996), is that this may be the result of a conflict between the 'reflexives first' condition (induced by the I-principle in our theory), which favours the use of a reflexive, and the locality condition, which goes against the use of a reflexive and therefore indirectly facilitates the use of a pronoun. Regardless 
of whether or not this explanation is on the right track, within the proposed revised neo-Gricean pragmatic framework, (8)-(10) and (31)-(36) can be analysed along the following lines. For reference, both the reflexive and the pronoun are subject to the (preferred) Iimplicated coreference. ${ }^{7}$ However, since the grammar allows the unmarked pronoun to be used to encode coreference, the speaker will use it if such an interpretation is intended. This gives rise to the question as to why the marked reflexive can also be used. Put another way, a question may be raised as to whether or not there is any systematic semantic/pragmatic contrast between the reflexive on the one hand, and the pronoun on the other. The answer is certainly yes. Intuitively, the use of a reflexive in these locations indicates some sort of unexpectedness (Edmondson and Plank 1978). Examined in a more careful way, this unexpectedness turns out to be emphatic-

${ }^{7}$ For the philosophical evidence (from Bar-Hillel, Carnap, and Popper) in support of the argument that a local coreferential interpretation is semantically more specific and informationally richer than, therefore preferred to, a local noncoreferential interpretation, see Huang (2000: 216). Note that such an interpretation, being a conversational implicature, is subject to defeasibility. For example, in (1), the I-implicated local coreferential interpretation is cancelled by the lexical semantics of the predicate, and in (2), it is overridden by our real world knowledge of which diseases can be transmitted by contact between people (contrast (3)).

(1) Chinese

Xiaohua $_{1}$ mai le $\mathrm{ta}_{2}$ de shu.

Xiaohua buy Pf $3 \mathrm{Sg}$ of book

"Xiaohuav ${ }_{1}$ bought his 2 book."

(2) David 1 has his ${ }_{2}$ flu.

(3) $*$ David $_{1}$ has his 2 ulcer.

All this indicates that given that conversational implicatures are cancelable, we can always arrive at an interpretation of coreferential possessive anaphora that is best in keeping with our knowledge about the world (see e.g., Huang 1991, 1994, 2000a for detailed discussion). 
50 Typology of Coreferential Possessive Anaphora and Neo-Gricean Pragmatics

ness/contrastiveness.

The use of an emphatic is in general subject to certain semantico-pragmatic conditions, such as those proposed by Baker (1995), and typically produces a number of effects: (i) contrariety to expectation, (ii) availability of a natural negative gloss, of the sort 'and not anyone else' etc., (iii) inducing a particular anaphoric/referential interpretation, (iv) contrastive stress, and (v) giving rise to a particular scope reading (e.g., Edmondson and Plank 1978, and especially Levinson 1991). Consider now (37).

(37) (Chinese)

Xiaohua $_{1}$ xihuan $\operatorname{ta}_{1 / 2} /$ ziji $_{1} /$ taziji $_{1} \quad$ de

Xiaohua like $3 \mathrm{Sg} / \mathrm{self} / 3 \mathrm{Sg}$ self of

youeryuan, bu xihuan didi de

nursery not like younger brother of

youeryuan.

nursery

"Xiaohua likes his $_{1 / 2} /$ self's $_{1} /$ his self's ${ }_{1}$ nursery, but does not like (his) brother's nursery."

The introduction of didi (younger brother) into the domain of discourse here is a clear indication that (37) conveys an emphatic/contrastive message. This seems to explain why intuitively, the use of ziji and taziji sound slightly more natural than $t a$ on the indexed interpretation. Furthermore, taziji is intuitively felt to be more emphatic/contrastive than ziji. On our account, the emphaticness/contrastiveness associated with the use of an emphatic reflexive falls out naturally of the M-principle: it is because the use of a reflexive in these contexts would carry an emphatic/contrastive message that would not be conveyed by the use of a pronoun that it is chosen. Furthermore, the fact that the use of taziji is more emphatic/contrastive than that of ziji can also be explained by the Mprinciple. Given this principle, it is predicted that the use of a more 
prolix expression tends to give a more marked message, hence a more emphatic/contrastive reading for taziji. Looked at from a slightly different vantage point, an iconicity principle is also in operation here, namely, the more coding material, the more emphatic/contrastive the message. This is exactly what happens with the use of 'both reflexives and pronouns' coreferential possessive anaphora in (8)-(10) and (31)-(36) above. ${ }^{8}$

\section{Implications for a Newly Designed Artificial Language}

What, then, are the implications of the typology and revised neo-Gricean pragmatic analysis, of coreferential possessive anaphora in natural languages, discussed above, for the construction of such an anaphoric device in a newly designed artificial language? Before we provide an answer to this question, let us first take a look at coreferential possessive anaphora in an existing artificial language, namely Esperanto. Simply put, coreferential possessive anaphora in Esperanto behaves like that in a 'reflexives only' language. This can be illustrated by a consideration of (38).

(38) (Esperanto)

Johano $_{1}$ amas $\operatorname{sian}_{1} /$ lian $_{2}$ edzinon.

John-Nom loves self's/his-Acc wife-Acc

"John ${ }_{1}$ loves self's $\mathrm{s}_{1} / \mathrm{his}_{2}$ wife."

Here, if the reflexive sia is used, we have a coreferential interpretation. On the other hand, if the pronoun lia is employed, we

${ }^{8}$ In fact, the repetition of the proper name in the Chinese example in note 1 above may also be given a similar account, for it also seems to carry an emphatic/contrastive message " . . but not any people else's". 
have a non-coreferential reading, thus the 'reflexives only' pattern.

Now, on the assumption that an artificial language should be a better instrument of communication than a natural language, it should be of maximum clarity. In other words, an artificial language should avoid ambiguity that is so pervasive in natural languages as much as possible. This holds, of course, for coreferential possessive anaphora in an artificial language. Thus, in working out coreferential possessive anaphora in a newly designed artificial language, two goals need to be achieved: firstly and most importantly to maximize clarity and minimize ambiguity, and secondly to cover other semantic aspects such as conveying an emphatic/contrastive message. Measured against these two goals, the 'pronouns only' model has to be rejected. This is because this mechanism is both ambiguous and inadequate in expressing emphasis/contrastiveness. Next, how about the 'both reflexives and pronouns' model? While it can convey an emphatic/contrastive message successfully, this model is ambiguous, therefore it has to be ruled out as the basis on which to design coreferential possessive anaphora in a newly constructed artificial language as well. This brings us to the final, 'reflexives only' model. Here the reverse is true: no ambiguity will arise, but no emphasis/contrastiveness can be conveyed either. However, given that, from a communicative point of view, clarity is more important than emphasis/contrastiveness, the 'reflexives only' model is better suited for our purposes than the 'both reflexives and pronouns' model. Therefore, we would use it as the basic model, retaining its 'avoid ambiguity' property, as in Esperanto while borrowing the emphasis/contrastiveness conveying part from the 'both reflexives and pronouns' model. Consequently, we shall propose that coreferential possessive anaphora in a newly designed artificial language $A$ should be constructed along the following lines: (i) a morphologically simplex reflexive $R$, which encodes coreferentiality between the possessive anaphor and its antecedent, (ii) a pronoun $P$, which indicates non-coreferentiality between the possessive anaphor and 
its antecedent, and (iii) a morphologically complex reflexive in the form of 'pronoun + reflexive', $P R$, which serves to convey emphasis/contrastiveness. This proposed mechanism for coreferential possessive anaphora in a newly designed artificial language can be illustrated by English glosses in (39).

(39) Coreferential possessive anaphora in a newly designed artificial language

a. Coreferential $R$

$\mathrm{John}_{1}$ likes self's, laptop.

b. Non-coreferential $P$ John ${ }_{1}$ likes his 2 laptop.

c. Coreferential and emphatic/contrastiveness $P R$ John likes his self's laptop.

\section{Summary}

In this article, I have presented a typology, and a revised neoGricean pragmatic analysis, of coreferential possessive anaphora in a range of genetically different and structurally diverse natural languages. I have also considered coreferential possessive anaphora in Esperanto and discussed how to construct such anaphora for a newly designed artificial language, combining the strengths of both the 'reflexives only' and 'both reflexives and pronouns' models in natural languages.

\section{References}

Amritavali, R. 2000. Lexical Anaphors and Pronouns in Kannada. In B. Lust et al (eds.), 49-112. 
54 Typology of Coreferential Possessive Anaphora and Neo-Gricean Pragmatics

Annamalai, E. 2000. Lexical Anaphors and Pronouns in Tamil. In B. Lust et al (eds.), 169-216.

Baker, C. 1995. Contrast, Discourse Prominence, and Intensification with Special Reference to Locally Free Reflexives in British English. Language 71, 63-101.

Biloa, E. 1991. Anaphora and Binding. Linguistics 29, 845-859.

Breeze, M. 1986. Personal Pronouns in Gimira. In U. Wiesemann (ed.), 47-69.

Burzio, L. 1991. The Morphological Basis of Anaphora. Journal of Linguistics 27, 81-105.

Burzio, L. 1996. The Role of The Antecedent in Anaphoric Relations. In R. Fredin (ed.), Current Issues in Comparative Grammar 1-45. Dordrecht: Kluwer.

Chomsky, N. 1981. Lectures on Government and Binding. Dordrecht: Foris.

Chomsky, N. 1995. The Minimalist Program. Cambridge, MA: MIT Press.

Davison, A. 1997. Lexical Anaphora in Hindi/Urdu. Ms., Iowa City, IO: University of Iowa.

Edmondson, J. \& F. Plank. 1978. Great Expectations: An Intensive Self Analysis. Linguistics and Philosophy 2, 373-413.

Faltz, L. 1985. Reflexivization: A Study in Universal Syntax. New York: Garland.

Farmer, A. \& R. Hanish. 1987. Communicative Reference with Pronouns. In J. Verschueren \& M. Bertuccelli-Papi (eds.), The Pragmatics Perspective 547- 565. Amsterdam: John Benjamins.

Grice, H. 1989. Studies in the Way of Words. Cambridge, MA: Harvard University Press.

Hestvik, A. 1992. LF Movement of Pronouns and Antisubject Orientation. Linguistic Inquiry 23, 557-594.

Huang, Y. 1991. A Neo-Gricean Pragmatic Theory of Anaphora. Journal of Linguistics 27, 301-335.

Huang, Y. 1994. The Syntax and Pragmatics of Anaphora: A Study 
with Special Reference to Chinese. Cambridge: Cambridge University Press.

Huang, Y. 1995. On Null Subjects and Null Objects in Generative Grammar. Linguistics 33, 1081-1123.

Huang, Y. 1996. A Note on the Head-movement Analysis of Longdistance Reflexives. Linguistics 34, 833-840.

Huang, Y. 2000a. Anaphora: A Cross-linguistic Study. Oxford: Oxford University Press.

Huang, Y. 2000b. Discourse Anaphora: Four Theoretical Models. Journal of Pragmatics 32, 151-176.

Huang, Y. In preparation. Pragmatics. To be published by Oxford: Oxford University Press.

Kitagawa, C. 1982. Topic Constructions in Japanese. Lingua 57, 175-214.

Levinson, S. 1987. Pragmatics and the Grammar of Anaphora. Journal of Linguistics 23, 379-434.

Levinson, S. 1991. Pragmatic Reduction of the Binding Conditions Revisited. Journal of Linguistics 27, 107-161.

Levinson, S. 2000. Presumptive Meanings: The Theory of Generalized Conversational Implicature. Cambridge, MA: MIT Press.

Lust, B., K. Wali, J. Gair, \& K. Subbarao. (eds.). 2000. Lexical Anaphors and Pronouns in Selected South Asian Languages. Berlin: Mouton de Gruyter.

Mohanan, K. 1983. Grammatical Relations and Anaphora in Malayalam. MIT Working Papers in Linguistics 163-190.

Nicholas, J. 2001. Long-distance Reflexivization in Chechen and Ingush. In P. Cole, G. Hermon, \& C. Huang (eds.), Longdistance Reflexivization 255-278. New York: Academic Press.

Parker, E. 1986. Mundani Pronouns. In U. Wiesemann (ed.), 131166.

Ray, T. 2000. Lexical Anaphors and Pronouns in Oriy. In B. Lust et al (eds.), 575-636.

Rebuschi, G. 1987. Defining the Three Binding Domains of Basque. 
56 Typology of Coreferential Possessive Anaphora and Neo-Gricean Pragmatics

Paper Presented in the Conference on the Basque Languages.

Sengupta, G. 2000. Lexical Anaphors and Pronouns in Bangala. In B. Lust et al (eds.), 277-332.

Shibatani, M. 1990. The Languages of Japan. Cambridge: Cambridge University Press.

Subbarao, K. \& B. Murthy. 2000. Lexical Anaphors and Pronouns in Telugu. In B. Lust et al (eds.), 217-276.

Wali, K. 2000. Lexical Anaphors and Pronouns in Marathi. In B. Lust et al (eds.), 513-574.

Wali, K., O. Koul, P. Hook, \& A. Koul. 2000. Lexical Anaphors and Pronouns in Kashmiri. In Lust et al (eds.), 471-512.

Wiesemann, U. (ed.). 1986. Pronominal Systems. Tübingen: Narr. 